\title{
Introduction to Gamification Minitrack
}

\author{
Juho Hamari \\ Gamification Group \\ Tampere University of Technology, \\ University of Turku, \\ \& University of Tampere \\ juho.hamari@tut.fi
}

\author{
Petri Parvinen \\ Mixed Reality Hub \\ University of Helsinki \\ petri.parvinen@helsinki.fi
}

Gamification refers to the ways in which our lives, and wider reality, are gradually becoming more game-like. It manifests not only as a pervasive, underlying development of our culture and society, but also as an intentional design process where services, systems and organizational structures are increasingly orchestrated to produce similar positive experiences to those offered by games.

Gamification has become a prominent vein of research during the last five years in HICSS-related fields such as information systems, computer science and business sciences. So too has the Gamification minitrack: since its inception it has grown into one of the largest minitracks of the conference in just a couple of years. The Gamification minitrack attracts research papers from a vast array of perspectives, and therefore, unites many of the different veins of research appearing throughout the HICSS conference. For HICSS2018, the track has a relatively larger focus on business compared to preceding years, due to our association with the SI call on Gamification at the prolific Journal of Business Research.

At HICSS2018, the Gamification minitrack includes such themes related to gamification as production, user/consumer experience and behavior, decision making, mixed reality, physical activity as well as the design and wider theory of gamification. The presentations include empirical studies, reviews of prior literature as well as more theoretical treatises on gamification.

The Gamification minitrack consists of 14 presentations based on the following papers:

"Gamification of the work floor: A literature review of gamifying production and logistics operations" by Harald Warmelink, Jonna Koivisto, Igor Mayer, Mikko Vesa and Juho Hamari

"A Theory of Gamification Principles Through Goal-Setting Theory" by Gustavo Tondello, Hardy Premsukh and Lennart Nacke
"Tailoring a Points Scoring Mechanism for Crowd-Based Knowledge Pooling" by Ganit Richter, Daphne R. Raban and Sheizaf Rafaeli

"What Drives Decision Makers to Follow or Ignore Forecasting Tools - A Game Based Analysis" by Georg Vetter, Markus Siepermann and Richard Lackes

"Points for Posts and Badges to Brand Advocates: The Role of Gamification in Consumer Brand Engagement" by Elena Vitkauskaitè and Rimantas Gatautis

"Gamified Workshops as Drivers for Attitudinal and Behavioral Shifts toward Sustainable Business Practices: The Role of Enjoyment, Curiosity and External Regulation" by Lisa-Maria Putz and Horst Treiblmaier

"Value Co-Creation and Co-Destruction in Online Video Games: An Exploratory Study and Implications for Future Research" by Jarkko Kokko, Tero Vartiainen and Tuure Tuunanen

"Employing Mixed Reality Applications: Customer Experience Perspective" by Jani Holopainen, Osmo Mattila, Petri Parvinen, Essi Pöyry and Kaapo Seppälä

"The Interaction of Trait Competitiveness and Leaderboard Design - An Experimental Analysis of Effects on Perceptions and Usage Intention" by Christoph E. Höllig, Andranik Tumasjan and Isabell M. Welpe

"Gamified Digital Services: How Gameful Experiences Drive Continued Service Usage" by Tobias Wolf, Welf H. Weiger and Maik Hammerschmidt

"Gamification of Older Adults' Physical Activity: An Eight-Week Study" by Dennis Kappen, Pejman Mirza-Babaei and Lennart Nacke

"What's in the Game? Developing a Taxonomy of Gamification Concepts for Health Apps" by Manuel Schmidt-Kraepelin, Scott Thiebes, Minh Chau Tran and Ali Sunyaev

"A Design Framework for Adaptive Gamification Applications" by Martin Böckle, Isabel Micheel, Markus Bick and Jasminko Novak 
"Beyond Enjoyment: A Cognitive-Emotional Perspective of Gamification" by Jeffrey K. Mullin and, Rajiv Sabherwal 